\title{
Initiating Opioid Use Disorder Medication via Telemedicine During COVID-19: Implications for Proposed Reforms to the Ryan Haight Act
}

\author{
Haiden A. Huskamp, Ph.D. ${ }^{7}$ (1), Lauren Riedel, M.P.H. ${ }^{7}$, Lori Uscher-Pines, Ph.D. ${ }^{2}$, \\ Alisa B. Busch, M.D., M.S. ${ }^{1,3}$, Michael L. Barnett, M.D., M.S. ${ }^{4,5}$, \\ Pushpa Raja, M.D., M.S.H.P.M' , and Ateev Mehrotra, M.D., M.P.H. ${ }^{1.7}$
}

\begin{abstract}
'Department of Health Care Policy, Harvard Medical School, 180A Longwood Avenue, Boston, MA, USA; ${ }^{2}$ RAND Corporation, Arlington, VA, USA; ${ }^{3}$ McLean Hospital, Belmont, MA, USA; ' ${ }^{4}$ epartment of Policy and Management, Harvard T.H. Chan School of Public Health, Boston, MA, USA; ${ }^{5}$ Division of General Internal Medicine and Primary Care, Department of Medicine, Brigham and Women's Hospital, Boston, MA, USA; ${ }^{6}$ Greater Los Angeles VA Medical Center, Los Angeles, CA, USA; ${ }^{7}$ Division of General Medicine, Beth Israel Deaconess Medical Center, Boston, MA, USA.
\end{abstract}

BACKGROUND: The Ryan Haight Act generally requires a clinician to conduct an in-person visit before prescribing an opioid use disorder (OUD) medication. This requirement has impeded use of telemedicine to expand OUD treatment, and many policymakers have called for its removal. During the COVID-19 pandemic, beginning March 16, 2020, the requirement was temporarily waived. It is unclear whether clinicians who treat OUD patients perceive telemedicine to be a safe and effective means of OUD medication initiation.

OBJECTIVE: To understand clinician use of and comfort level with using telemedicine to initiate patients on medication for opioid use disorder.

DESIGN: National survey administered electronically via WebMD/Medscape's online clinician panel in fall 2020.

PARTICIPANTS: A total of 602 clinicians (primary care providers, psychiatrists, nurse practitioners or certified nurse specialists, and physician assistants) participated in the survey.

MAIN MEASURES: Frequency of video, audio-only, and in-person visits for medication initiation, comfort level with using video for new patient visits with OUD.

KEY RESULTS: Clinicians varied substantially in their use of telemedicine for medication initiation. Approximately $25 \%$ used telemedicine for most initiations while $40 \%$ used only in-person visits. The majority (55.8\%) expressed at least some discomfort with using telemedicine for treating new OUD patients, although clinicians with more OUD patients were less likely to express such discomfort.

CONCLUSION: Findings suggest that a permanent relaxation of the Ryan Haight requirement may not result in widespread adoption of telemedicine for OUD medication initiation without additional supports or incentives.

KEY WORDS: telemedicine; behavioral health; substance use disorders.

J Gen Intern Med 37(1):162-7

DOI: $10.1007 / \mathrm{s} 11606-021-07174-w$

(C) Society of General Internal Medicine 2021

Received May 26, 2021

Accepted September 27, 2021

Published online October 28, 2021

\section{INTRODUCTION}

Medications such as buprenorphine are highly effective for the treatment of opioid use disorder (OUD), yet most individuals with OUD do not receive these medications. ${ }^{1,2}$ An important barrier to OUD medication treatment is a shortage of prescribing providers, particularly in rural and other underserved areas. ${ }^{2}$ Telemedicine could improve access to OUD treatment. However, the 2008 Ryan Haight Online Pharmacy Consumer Protection Act, passed to prevent online pharmacies from directly dispensing controlled substances without adequate medical evaluation, ${ }^{3}$ is a major obstacle. The Act generally requires that clinicians conduct an in-person visit before prescribing controlled substances, including OUD medications such as buprenorphine. The requirement of an in-person visit could lead to delays in initiating care and may be particularly problematic for rural residents for whom travel distances to care may be long, for individuals with other barriers such as unreliable transportation or work-related or child-care issues, and for those wrestling with perceived stigma of presenting to in-person clinics. The in-person requirement also impedes the operations and growth of telehealth companies treating patients with OUD, ${ }^{4}$ requiring them to have a physical footprint in every community they serve.

During the COVID-19 emergency, both patients and clinicians feared exposure to the virus during in-person visits. In response to the pandemic, the Ryan Haight Act's in-person visit requirement was temporarily waived in March of 2020. Through the end of the public health emergency, clinicians can prescribe medication treatment via either video or audio-only (i.e., telephone) visits. This temporary waiver creates a unique opportunity to learn from clinicians' experiences with using telemedicine for OUD medication initiation and treatment in the absence of the previous restrictions. This is particularly relevant given that the SUPPORT for Patients and Communities Act, passed in 2018, called for the creation of a pathway for clinicians to register with the Drug Enforcement 
Administration (DEA) to provide telemedicine OUD services and allow registered providers to bypass the in-person requirement when prescribing medications such as buprenorphine. ${ }^{3,5,6}$ The legislation called for this pathway to be created by October 2019; however, this pathway still has not been implemented.

It is unclear whether clinicians who treat individuals with OUD perceive telemedicine to be a safe and effective means of OUD medication initiation. To explore clinician perspectives, we conducted the first national survey of clinicians who treat individuals with OUD about their use and comfort level with telemedicine for OUD medication initiation during the pandemic.

\section{METHODS}

We administered an electronic survey between late November and mid-December 2020 to a national sample of clinicians who treat individuals with OUD. Participants were recruited using WebMD/Medscape's online panel of 2.5 million clinicians. This panel is composed of clinicians who have joined the platform to access clinical content and tools as well as continuing medical education activities. ${ }^{7}$ The platform is commonly used for clinician recruitment in many federal studies including projects sponsored by the Centers for Disease Control (CDC) and by the Food and Drug Administration (FDA) to track clinicians' attitudes, behaviors, and practices ${ }^{8-10}$, as well as the impact of the COVID-19 pandemic on providers. ${ }^{11,12}$ To recruit participants for this survey, WebMD/ Medscape emailed a study invitation to all psychiatrists in the panel and a random sample of primary care physicians, nurse practitioners, and physician assistants. Clinicians who expressed interest were screened for eligibility (criteria described below) via an online instrument; if eligible, they were invited to complete the survey. Recruitment continued until a target sample size was reached $(n=600)$. There were 3816 clinicians who responded to the eligibility screener, of which, 602 met our entry criteria. Given the opt-in design of the survey, we were not able to calculate a response rate. However, the survey completion rate (the proportion of those who completed the survey among all respondents who passed screening criteria) was $97.3 \%$. Informed consent was obtained electronically. The survey was determined to be exempt by the Harvard Faculty of Medicine Institutional Review Board.

We hypothesized that a large proportion of OUD clinicians would initiate patients on OUD medications via telemedicine after the Ryan Haight Act restrictions were waived and that many would report being comfortable with using telemedicine for treating new OUD patients after having used the technology as of late fall 2020. Survey questions focused on clinician experiences with using telemedicine (defined in the survey as synchronous visits that occur either via audio-only or videoconferencing technology) to treat patients with OUD in the past month, which would refer to the period between
November and December 2020. (See Appendix for list of survey questions analyzed.) The survey included a ninequestion screener and 18 additional questions requesting information about clinician specialty and practice setting, characteristics of their patient caseload, whether they had initiated patients on OUD medications in the past month, their use of telemedicine when initiating OUD medications (both video and audio-only technology), and their comfort level in using telemedicine to provide OUD treatment services to new patients. To pilot test the survey, we first had four clinician researchers who treat OUD review the instrument and provide feedback to the study team. We then reviewed the draft survey instrument with five clinicians who met our entry criteria who were recruited by WebMD/Medscape. We asked the clinicians how they were interpreting the questions, whether any terms or questions were confusing, and whether there were any additional survey questions that they felt would be important to include based on their experiences.

\section{Inclusion/Exclusion Criteria}

Primary care physicians, psychiatrists, nurse practitioners or certified nurse specialists, and physician assistants were eligible to participate. Participating clinicians had to currently hold a buprenorphine waiver, spend at least $8 \mathrm{~h}$ per week practicing in an outpatient setting, prescribe OUD medications to two or more patients in a typical month, and report having used telemedicine with at least one OUD patient since the start of the pandemic. To focus on outpatient clinicians who were not primarily employed by a telehealth company, we excluded those who used telemedicine for $75 \%$ or more of OUD visits in 2019 as well as those whose main practice setting was an inpatient or residential treatment setting or an emergency department. Clinicians who were active-duty military or worked for Veterans Affairs (VA) or the Indian Health Service in their main practice setting were also excluded because these clinicians are subject to different reimbursement systems, and system-level policies governing telemedicine use.

\section{Data Analyses}

We used Stata v16 to conduct descriptive analyses of survey responses. Statistical significance was tested using chi-square tests. Because we hypothesized that clinician reports of their comfort level using video visits for new patients with OUD would differ for clinicians with a relatively high (vs. relatively low) proportion of OUD patients in their caseload, we compared clinician responses to this question across tertiles based on the proportion of OUD patients in their patient panel (i.e., high vs. medium vs. low relative share of caseload having OUD). To identify how many clinicians primarily used telemedicine for their OUD medication initiations, we also calculated the proportion of all clinicians using telemedicine for initiations who used telemedicine for the majority (i.e., $51 \%$ or more) of these initiation visits. 


\section{RESULTS}

Among the 602 survey respondents, 241 (40.0\%) were primary care physicians (PCPs), 141 were psychiatrists (23.4\%), $139(23.1 \%)$ were nurse practitioners or certified nurse specialists, and $81(13.5 \%)$ were physician assistants (Table 1). In terms of their primary practice setting, approximately threequarters practiced in either a single specialty group practice (236 or $39.2 \%$ ) or multi-specialty group practice (205 or $34.1 \%), 98(16.3 \%)$ were solo practitioners, 31 (5.2\%) worked in an opioid treatment program, and $32(5.3 \%)$ worked in other settings. Approximately one in ten (57 or 9.5\%) reported that their primary practice setting was located in a rural area, and respondents were located in 49 states.

Across the clinicians, the mean estimated percent of patients with various types of insurance was $34.4 \%$ private/ commercial insurance, $33.0 \%$ Medicaid, $19.6 \%$ Medicare, $9.0 \%$ uninsured, and $4.1 \%$ with another form of insurance. The mean (median) estimated percent of a clinician's patients with OUD was $27.8 \%$ (20.0\%). Just over half (351 or 58.3\%) did not use telemedicine for any patients with OUD in 2019.

\section{Initiation of OUD Medications}

Among the 602 surveyed clinicians, 506 (84.1\%) reported initiating at least one patient on OUD medication (either inperson or via telemedicine) during the past month. The characteristics of this large subset were similar to those of the full sample of respondents (Table 1).

Use of Telemedicine for Medication Initiation. There was considerable variation across clinicians in use of telemedicine for OUD medication initiation. Among the 506 who initiated OUD medication for at least one patient in the past month, most (303, or $59.9 \%$ ) used telemedicine for at least one of these visits, while two in five $(203$, or $40.1 \%)$ conducted medication initiation only via in-person visits. The characteristics of those who used telemedicine for medication initiation

Table 1. Characteristics of All OUD Clinicians Surveyed, Those Who Conducted Any OUD Medication Initiation Visits in the Past Month, and Those Who Used Telemedicine for at Least One Initiation Visit

\begin{tabular}{|c|c|c|c|}
\hline & $\begin{array}{l}\text { Overall sample } \\
(n=602)\end{array}$ & $\begin{array}{l}\text { Clinicians who } \\
\text { performed } \\
\text { at least one initiation } \\
\text { visit } \\
\text { in past month } \\
(n=506)\end{array}$ & $\begin{array}{l}\text { Clinicians who used } \\
\text { telemedicine } \\
\text { for at least one initiation } \\
\text { visit in past month } \\
(N=303)\end{array}$ \\
\hline \multicolumn{4}{|l|}{ Training, $N(\%)$} \\
\hline Primary care physician & $241(40.0 \%)$ & $207(40.9 \%)$ & $128(42.2 \%)$ \\
\hline Psychiatrist & $141(23.4 \%)$ & $114(22.5 \%)$ & $75(24.8 \%)$ \\
\hline Nurse Practitioner & $139(23.1 \%)$ & $111(21.9 \%)$ & $67(22.1 \%)$ \\
\hline Physician Assistant & $81(13.5 \%)$ & $74(14.6 \%)$ & $33(10.9 \%)$ \\
\hline \multicolumn{4}{|l|}{ Type of practice, ${ }^{*} N(\%)$} \\
\hline Single specialty group practice & $236(39.2 \%)$ & $200(39.5 \%)$ & $119(39.3 \%)$ \\
\hline Multi-specialty group practice & $205(34.1 \%)$ & $177(35.0 \%)$ & $111(36.6 \%)$ \\
\hline Solo practice & $98(16.3 \%)$ & $75(14.8 \%)$ & $43(14.2 \%)$ \\
\hline Opioid treatment program (methadone program) & $31(5.2 \%)$ & $30(5.9 \%)$ & $17(5.6 \%)$ \\
\hline Other & $32(5.3 \%)$ & $24(4.7 \%)$ & $13(4.3 \%)$ \\
\hline \multicolumn{4}{|l|}{ Location of practice, $\dagger N(\%)$} \\
\hline Large city & $230(38.2 \%)$ & $195(38.5 \%)$ & $137(45.2 \%)$ \\
\hline Suburb near a large city & $223(37.0 \%)$ & $191(37.8 \%)$ & $110(36.3 \%)$ \\
\hline Small city or town & $92(15.3 \%)$ & $73(14.4 \%)$ & $35(11.6 \%)$ \\
\hline \multirow[t]{2}{*}{ Rural area } & $57(9.5 \%)$ & $47(9.3 \%)$ & $21(6.9 \%)$ \\
\hline & \multicolumn{3}{|c|}{ Years since completing medical training, $N(\%)$} \\
\hline Less than 5 years & $100(16.6 \%)$ & $89(17.6 \%)$ & $53(17.5 \%)$ \\
\hline $5-10$ years & $191(31.7 \%)$ & $159(31.4 \%)$ & $99(32.7 \%)$ \\
\hline $11-15$ years & $95(15.8 \%)$ & $77(15.2 \%)$ & $44(14.5 \%)$ \\
\hline 16 or more years & $216(35.9 \%)$ & $181(35.8 \%)$ & $107(35.3 \%)$ \\
\hline \multicolumn{4}{|l|}{ Use of telemedicine in 2019 for patients with OUD, $N(\%)$} \\
\hline No telemedicine in 2019 & $351(58.3 \%)$ & $286(56.5 \%)$ & $151(49.8 \%)$ \\
\hline $1-5 \%$ of visits & $89(14.8 \%)$ & $70(13.8 \%)$ & $41(13.5 \%)$ \\
\hline $6-30 \%$ of visits & $105(17.4 \%)$ & $99(19.6 \%)$ & $70(23.1 \%)$ \\
\hline $31-74 \%$ of visits & $57(9.5 \%)$ & $51(10.1 \%)$ & $41(13.5 \%)$ \\
\hline \multicolumn{4}{|l|}{ Patient insurance types, mean \% (standard deviation) } \\
\hline Private/commercial insurance & $34.4 \%(25.5)$ & $33.3 \%(24.6)$ & $33.7 \%(24.4)$ \\
\hline Medicaid & $33.0 \%(27.3)$ & $33.9 \%(27.2)$ & $33.7 \%(26.6)$ \\
\hline Medicare & $19.6 \%(16.8)$ & $19.8 \%(16.3)$ & $19.7 \%(15.3)$ \\
\hline Other & $4.1 \%(12.5)$ & $4.0 \%(11.9)$ & $3.8 \%(9.7)$ \\
\hline No insurance & $9.0 \%(15.9)$ & $9.0 \%(16.1)$ & $9.2 \%(16.3 \%)$ \\
\hline Mean percent of patients with OUD, mean \% (standard deviation) & $27.8 \%(27.4)$ & $29.7 \%(28.0)$ & $31.9 \%(28.2 \%)$ \\
\hline $\begin{array}{l}\text { Mean percent of Black/African American patients, mean \% } \\
\text { (standard deviation) }\end{array}$ & $20.8 \%(18.9)$ & $21.1 \%(18.8)$ & $23.5 \%(19.2)$ \\
\hline Mean percent of Latino patients, mean \% (standard deviation) & $17.9 \%(17.1)$ & $18.5 \%(17.6)$ & $19.9 \%(17.3)$ \\
\hline $\begin{array}{l}\text { Mean percent of patients who prefer a language other than } \\
\text { English, mean \% (standard deviation) }\end{array}$ & $15.1 \%(23.4)$ & $16.2 \%(24.6)$ & $17.6 \%(24.9)$ \\
\hline
\end{tabular}

*This row reflects the type of practice for the main practice setting reported by the respondent

This row reflects the location of practice for the main practice setting reported by the respondent 
were similar to the overall group of clinicians surveyed (Table 1).

Among the 506 clinicians who performed any medication initiation visits, on average, they used telemedicine for a third $(33.9 \%)$ of those visits versus $55.8 \%$ of all their OUD visits (i.e., not just those for medication initiation) (Fig. 1).

Among the 303 clinicians who used telemedicine for at least one medication initiation, $101(33.3 \%)$ used video for the majority (51\% or more) of the initiation visits they conducted and $23(7.6 \%)$ used telephone for the majority of their initiation visits. Among all 506 clinicians who conducted an initiation within the past month, 148 (or 29.2\%) used either video or audio-only telemedicine for the majority of their initiation visits.

Reported Level of Comfort Using Telemedicine for New OUD Patients. When asked how comfortable they were caring for new patients with OUD via video visits, approximately one-third of the 602 clinicians surveyed $(37.7 \%)$ reported being somewhat or very comfortable, while $55.8 \%$ reported being somewhat or very uncomfortable. Clinicians with more OUD patients were more likely to report being somewhat or very comfortable caring for new OUD patients via video ( $48.2 \%$ highest tertile, $43.0 \%$ middle tertile, $25.2 \%$ lowest tertile of volume, $p \leq 0.001$ ) (Figure 2).

\section{DISCUSSION}

There is considerable policy interest in using telemedicine to increase access to evidence-based OUD treatment. Much of the focus has been on the Ryan Haight Act provision requiring an in-person visit before prescribing buprenorphine or other OUD medications that are controlled substances. After Ryan Haight restrictions on prescribing OUD medications via telemedicine were temporarily relaxed during the COVID-19 pandemic, clinicians varied substantially in their use of telemedicine for the initiation of OUD medications. Almost 30\% (148 of 506) of clinicians used telemedicine (either video or audio-only) for the majority of their initiation visits while $40 \%$ (203 of 506) used only in-person visits (i.e., no telemedicine) for medication initiation. These disparate rates of use are consistent with reported levels of discomfort using telemedicine for treating new OUD patients during the pandemic. ${ }^{13} \mathrm{~A}$ small majority of OUD clinicians expressed at least some discomfort with the use of video visits for treating new patients with OUD, and clinicians were less likely to use telemedicine for medication initiation than for other aspects of OUD outpatient care.

Prior research has shown that some clinicians may be hesitant to use telemedicine for medication initiation visits due to liability or safety concerns. ${ }^{13}$ Others may have been hesitant to initiate patients on OUD medications during the pandemic because of reduced access to non-pharmacologic supports such as group therapy, peer support specialists, or support groups like Narcotics Anonymous, or they may not have been aware of the changes to the Ryan Haight Act requirements. Individual patient-level factors also likely influence the decision of whether to use telemedicine for medication initiation for a given patient. Nevertheless, our findings signal that a permanent relaxation of the Ryan Haight requirement may not result in universal adoption of telemedicine for OUD medication initiation without additional incentives and supports.

It is striking that clinicians with a relatively high percentage of patients with OUD in their caseload were more comfortable with using telemedicine to treat new OUD patients than those with a relatively low percentage. This could suggest that clinicians who are more experienced in treating OUD may be more likely to perceive telemedicine to be a safe and effective option and that the reluctance to use telemedicine among some may stem from less familiarity with OUD treatment in general. If so, this suggests a potential role for clear

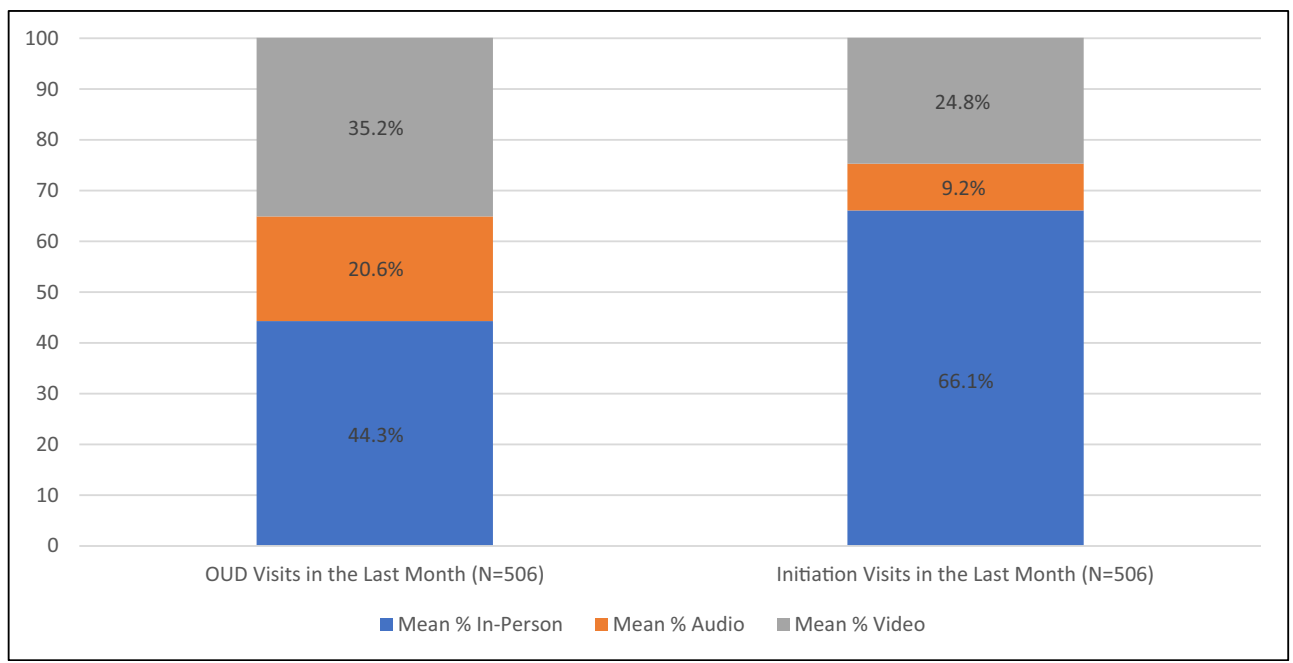

Figure 1. Mean clinician-reported percent of their OUD visits delivered in-person, via telephone, or via videoconference, for all OUD visits and for OUD medication visits only, among clinicians who conducted at least one initiation in the past month $(N=506)$ 


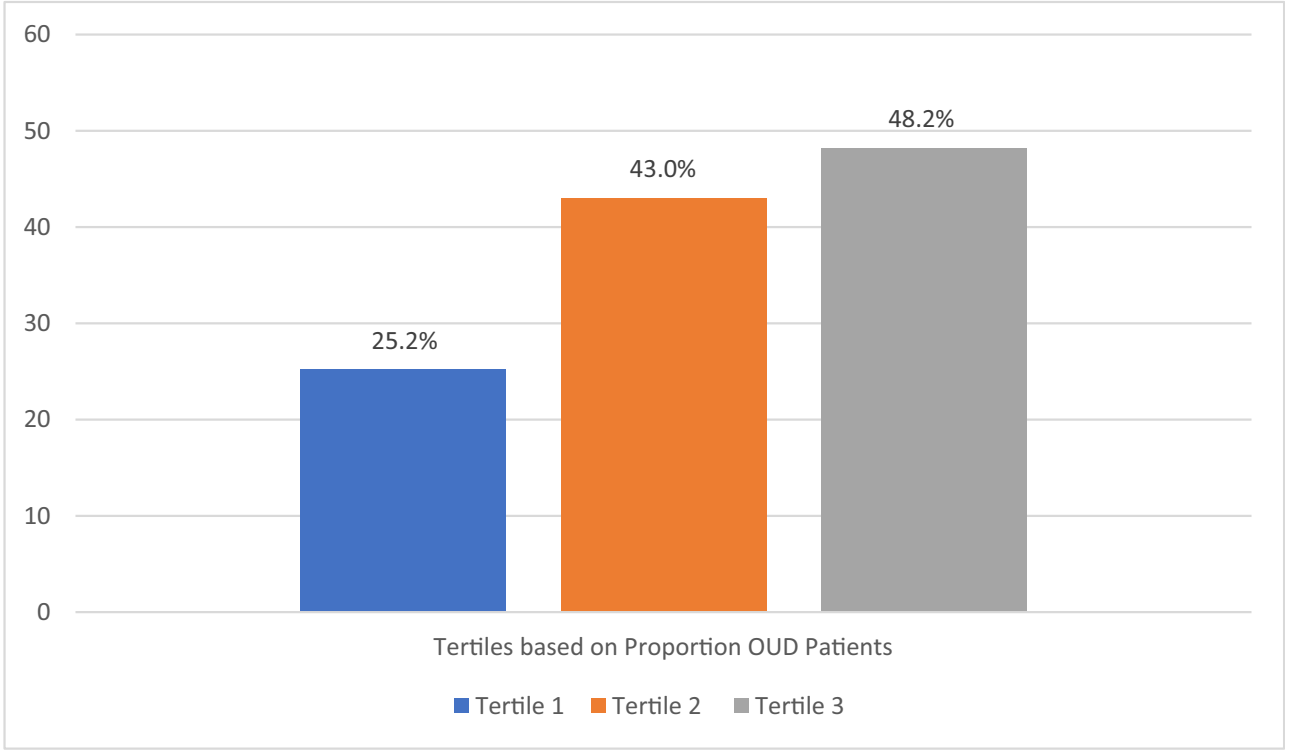

Figure 2. Percent of clinicians who report being somewhat or very comfortable treating new patients via video, by tertiles based on percent of OUD patients. Note: This shows the percent of clinicians who responded either "somewhat comfortable" or "very comfortable" in response to the prompt for "new patients" when asked the following question: "In the past month, how comfortable were you with caring for the following types of patients with opioid use disorder via video visits?" The $p$-value for difference between tertiles based on OUD patients is $\leq 0.001$. Tertile 3 includes those with the highest percentage of OUD patients in their caseload; tertile 1 includes those with the lowest.

guidelines on appropriate use of telemedicine for OUD medication initiation. Also, additional tools (e.g., home urine toxicology kits, peripheral devices that allow remote patient monitoring for vital signs or other in-person medical examination components) and treatment models (e.g., home visit by nurse, in-person visit $\mathrm{w} /$ clinic staff not attended by prescribing provider) might help more providers feel comfortable seeing new patients via telemedicine. Other forms of support could include expanding efforts within the Veterans Administration system that provide step-by-step guidance embedded within electronic medical records to help clinicians less comfortable with OUD treatment, provider-to-provider support such as Project Echo programs, and mentored training in the treatment of OUD via telemedicine during residency training.

Interestingly, while most clinicians who used telemedicine for at least one medication initiation used video visits, roughly $10 \%$ of their initiation visits were conducted via telephone, and a small subset of clinicians $(\sim 5 \%)$ reported using telephone for the majority of their medication initiation visits. Concerns exist that telephone visits may result in lower quality of care relative to video or in person. However, due to the "digital divide," many vulnerable patients may not have access to the technology needed for video visits (e.g., personal device, internet access), and telephone visits may be the only choice available for some to initiate treatment. Whether telephone visits lead to comparable care is a topic that merits further research.

Several limitations should be noted. First, the survey respondents were a non-probability-based sample of clinicians who deliver OUD medication treatment and therefore not generalized to all clinicians who treat OUD. However, as noted above, this clinician panel has been used in many other prior studies, including some by the DEA and Centers for
Disease Control and Prevention (CDC), ${ }^{8-10}$ and our sample of respondents was generally similar to prior national surveys of waivered clinicians in terms of clinician type, years since training, and rural/urban location ${ }^{14}$ (see Appendix for details). Second, clinicians were surveyed in November/December 2020, and most were relatively new to telemedicine at that point in time. Their responses to certain questions, such as the question about their comfort level using video technology for new patient visits, may evolve over time. Third, with this brief survey, we were unable to parse out all factors underlying clinician reported discomfort with the use of telemedicine for treating new patients with OUD. Finally, these exploratory analyses did not control for testing of multiple outcomes.

While the Ryan Haight Act requirement of an in-person visit stemmed from concerns about the distribution of controlled substances without proper medical evaluation and the potential for diversion, this requirement is a barrier to expanding access to OUD treatment via telemedicine, particularly in rural and other underserved areas. ${ }^{13,15}$ Our findings suggest that a more permanent waiver of the Ryan Haight requirement could potentially expand access to OUD care in a patient-centered way, allowing patients to engage in regular, timely OUD care without significant work/life balance disruptions and potentially alleviating some of the perceived stigma sometimes experienced in seeking in-person care. However, among clinicians who prescribe OUD medications, considerable hesitancy about the use of telemedicine for medication initiation remains. To expand access to OUD medication after the DEA's new pathway is introduced or permanent policy changes are made to waive the Ryan Haight requirement, further training and explicit guidelines for telemedicine use for OUD medication initiation may be needed to address this discomfort. 
Corresponding Author: Haiden A. Huskamp, Ph.D.; Department of Health Care Policy, Harvard Medical School, 180A Longwood Avenue, Boston, MA 02115, USA (e-mail: Huskamp@hcp.med.harvard.edu).

Supplementary Information The online version contains supplementary material available at https://doi.org/10.1007/s11606-02107174-w.

Funding This study was funded by NIDA (3R01DA048533-02S1). Drs. Huskamp and Busch were also supported by an additional grant from NIDA (P3O DA035772).

Declarations: Conflict of Interest

Dr. Barnett reports being retained as an expert witness for govern ment plaintiffs in lawsuits against opioid manufacturers and distributors. The authors have no other potential conflicts of interest to disclose.

\section{REFERENCES}

1. Substance Abuse and Mental Health Services Administration. Key Substance Use and Mental Health Indicators in the United States: Results from the 2019 National Survey on Drug Use and Health (HHS Publication No. PEP20-07-01001, NSDUH Series H-55). Rockville: Center for Behavioral Health Statistics and Quality, Substance Abuse and Mental Health Services Administration. Retrieved from https://www.samhsa.gov/data/;2020.

2. National Academies of Sciences E, Medicine. Medications for Opioid Use Disorder Save Lives. Washington: The National Academies Press; 2019.

3. Dooling BCE, Stanley L. Extending Pandemic Flexibilities for Opioid Use Disorder Treatment: Telemedicine \& Initiating Buprenorphine Treatment. 2021; https://regulatorystudies.columbian.gwu.edu/extending-pandemic-flexibilities-opioid-use-disorder-treatment. Accessed May 11, 2021.
4. Uscher-Pines L, Huskamp HA, Mehrotra A. Treating Patients With Opioid Use Disorder in Their Homes: An Emerging Treatment Model. JAMA. 2020;324(1):39-40.

5. Davis CS. The SUPPORT for Patients and Communities Act - What Will It Mean for the Opioid-Overdose Crisis? N Engl J Med. 2019;380(1):3-5

6. Congress.gov. H.R.6 - SUPPORT for Patients and Communities Act, 115th Congress (2017-2018). https://www.congress.gov/bill/115th-congress/ house-bill/6. Accessed August 11, 2021.

7. WebMD Medscape. Market Research 2020; https://www.medscape.com/ sites/public/marketresearch. Accessed May 11, 2021.

8. Mezher M. FDA to Survey Healthcare Professionals on Prescription Drug Marketing. 2019; https://www.raps.org/news-and-articles/news-articles /2019/3/fda-to-survey-healthcare-professionals-on-prescrip. Accessed May 11, 2021.

9. U.S. Food and Drug Administration. Office of Pharmaceutical Quality: 2019 Annual Report February 2020.

10. Carla L. Black, Xin Yue, Sarah W. Ball, et al. Influenza Vaccination Coverage Among Health Care Personnel - United States, 2017-18 Influenza Season. MMWR Morb Mortal Wkly Rep. 2018;67:1050-1054.

11. Locke T. Nearly 1 in 3 on Front Line Lacked PPE: Medscape UK COVID19 Poll. Medscape. May 19, 2020. https://www.medscape.com/ viewarticle/930736. Accessed 11 May 2021

12. Uscher-Pines L, Sousa J, Raja P, Mehrotra A, Barnett ML, Huskamp HA. Suddenly Becoming a "Virtual Doctor": Experiences of Psychiatrists Transitioning to Telemedicine During the COVID-19 Pandemic. Psychiatr Serv. 2020;71(11):1143-1150.

13. Uscher-Pines L, Sousa J, Raja P, Mehrotra A, Barnett M, Huskamp HA. Treatment of opioid use disorder during COVID-19: Experiences of clinicians transitioning to telemedicine. J Subst Abuse Treat. 2020;118:108124.

14. Jones CM, McCance-Katz EF. Characteristics and prescribing practices of clinicians recently waivered to prescribe buprenorphine for the treatment of opioid use disorder. Addiction. 2019;114(3):471-482.

15. Davis CS, Samuels EA. Continuing increased access to buprenorphine in the United States via telemedicine after COVID-19. Int J Drug Policy. 2020:93: 102905 .

Publisher's Note: Springer Nature remains neutral with regard to jurisdictional claims in published maps and institutional affiliations. 